\title{
Disgust and implicit self-concept in women with borderline personality disorder and posttraumatic stress disorder
}

\author{
Nicolas Rüsch • Daniela Schulz • Gabi Valerius • \\ Regina Steil · Martin Bohus · Christian Schmahl
}

Received: 21 July 2010/ Accepted: 19 November 2010/Published online: 5 December 2010

(C) Springer-Verlag 2010

\begin{abstract}
Disgust may be a key emotion and target for psychotherapeutic interventions in borderline personality disorder (BPD) and posttraumatic stress disorder (PTSD) at explicit and implicit-automatic levels. However, automatically activated disgust reactions in individuals with these disorders have not been studied. Disgust and its correlation with childhood abuse were assessed in women with BPD, but without PTSD; women with PTSD, but without BPD; women with BPD and PTSD; and healthy women. Disgust sensitivity, anxiety and depression were measured by selfreport. Implicit disgust-prone (relative to anxiety-prone) self-concept was assessed using the Implicit Association Test. Women with BPD and/or PTSD reported more disgust sensitivity than controls. The implicit self-concept among patients was more disgust-prone (relative to anxietyprone) than in controls. Women with BPD, with PTSD, or BPD and PTSD did not differ significantly in self-reported disgust levels or implicit disgust-related self-concept. Among women with BPD and/or PTSD, current psychiatric comorbidity (major depression, anxiety disorder, eating disorder, or substance-related disorder) did not affect disgust-related variables. More severe physical abuse in childhood was associated with a more anxiety-prone
\end{abstract}

\footnotetext{
N. Rüsch ( $\square)$

Department of Social and General Psychiatry,

Psychiatric University Hospital Zürich,

Militärstr. 8/Postfach 1930, 8021 Zürich, Switzerland

e-mail: nicolas.ruesch@dgsp.uzh.ch

N. Rüsch

Department of Psychiatry and Psychotherapy,

University of Freiburg, Freiburg, Germany

D. Schulz · G. Valerius - R. Steil - M. Bohus - C. Schmahl Department of Psychosomatic Medicine and Psychotherapy, Central Institute of Mental Health, Mannheim, Germany
}

(less disgust-prone) implicit self-concept. Independent of psychiatric comorbidity, disgust appears to be elevated at implicit and explicit levels in trauma-related disorders. Psychotherapeutic approaches to address disgust should take implicit processes into account.

Keywords Disgust - Borderline personality disorder . Posttraumatic stress disorder - Implicit association test

\section{Introduction}

After being neglected in psychiatric research for decades $[28,31]$, disgust more recently was found to be associated with phobic and obsessive-compulsive $[27,29,33,40]$ as well as eating disorders [12, 43] and schizophrenia [36]. Clinical experience and theoretical models $[10,21]$ further suggest that disgust is a key emotion in conditions with high levels of exposure to traumatic stress. Sexually traumatized individuals frequently report disgust in relation to food, fluids associated with traumatic events, or to their own body. Regarding posttraumatic stress disorder (PTSD [7, 23]), Dalgleish and Power [10] argued that disgust can be the central emotion in this disorder and lead to selfdisgust. So far, however, there is little empirical evidence to support this claim. An early report [15], using a problem checklist, found disgust to be common in veterans with PTSD. As far as borderline personality disorder (BPD) is concerned, women with BPD facially expressed increased disgust during an attachment interview in a recent study [6], possibly linked to memories of childhood abuse that are common in BPD [44]. This is consistent with the view that emotional dysregulation, a central feature in BPD for emotions such as anxiety, anger or shame [25, 34], extends to disgust. We therefore set out to study disgust in women 
with BPD and in women with PTSD and a history of sexual traumatization. The inclusion of women with only BPD, only PTSD, and both BPD and PTSD allowed us to examine possible additive effects of both disorders on disgust levels.

Disgust, literally 'bad taste', has been defined [11] as referring "to something revolting, primarily in relation to the sense of taste, as actually perceived or vividly imagined; and secondarily to anything which causes a similar feeling, through the sense of smell, touch and even of eyesight" (p. 253). Disgust may operate at explicit levels, accessible by self-report measures, as well as at more automatically activated, implicit levels that can be assessed by response-latency measures such as the Implicit Association Test (described below). A growing literature has shown that more rapid, automatic reactions, which may operate outside conscious awareness and control, can predict behaviours independently of more deliberate, explicit self-reported beliefs [16, 20]. This distinction also applies to a person's self-concept which is characterized both by explicit, consciously accessible and by implicit-automatic cognitions that are not necessarily congruent. For example, a person with a disgust-prone self-concept may explicitly say 'I am disgusting', a statement which is at an implicit level usually accompanied by a variety of affects and memories that the individual may not be aware of. In our study, an implicit-automatic disgust-prone self-concept implies that the concept of Self can automatically activate disgust if these concepts are associated with one another in memory. This automatic activation of disgust can occur whether or not the participant deliberately endorses the proposition to be disgusting her- or himself and whether or not disgust in relation to oneself is consciously experienced by the person. This view of automaticity of emotion is consistent with recent work on implicit or unconscious aspects of emotional reactions $[1,13,35]$. We expected the implicit disgust-prone self-concept to be modestly positively correlated with self-reported disgust (if at all; see [22]).

Cognitive-behavioural [2] or psychodynamic [21] researchers would refer to implicit-automatic processes as schemata or unconscious memories, respectively, that often guide our perceptions and behaviour. It is therefore of high clinical relevance to examine not only explicit, selfreported, but also implicit, automatically activated aspects of disgust in order to assess its full impact in trauma-related disorders. Automatically activated pathological disgust in trauma-related disorders would need to be treated differently from self-reported disgust [16].

As far as explicit measures are concerned, a recent study found self-disgust to be related to depression [30]. With respect to implicit-automatic disgust, initial evidence from healthy individuals suggests that disgust affects automatic cognitive processing [8, 9]. We used a well-established reaction-time task, the Implicit Association Test (IAT [18]), as a measure of a disgust-prone implicit self-concept. The IAT uses response latencies to determine implicit associations between concepts and attributes (e.g., Self and Disgust), based on the notion that quicker processing speeds equate with stronger associations. It has been used to measure implicit self-concept $[17,34]$ and dysfunctional cognitive schemata, especially in anxiety disorders [41]. The IAT can measure implicit associations that a person may not be aware of or may not be willing to disclose in self-report measures. This is an important advantage in the assessment of aversive emotions such as disgust, reducing the influence of self-presentational strategies and social desirability concerns.

Our study was designed to test the hypotheses that first, self-reported disgust sensitivity is higher in women with BPD and/or PTSD when compared to healthy women; and second that women with BPD and/or PTSD have a more disgust-prone implicit self-concept than healthy women, as evidenced by a stronger association of self with disgust (relative to anxiety) during the IAT. Because sexual abuse in childhood is likely to be associated with disgust, our third hypothesis was that greater severity of childhood sexual abuse would be related to more self-reported disgust sensitivity and to a more disgust-prone implicit selfconcept.

\section{Method}

\section{Participants}

Twenty women with BPD, but without PTSD, 20 women with PTSD, but without BPD, and 15 women with BPD and PTSD were recruited at the Department of Psychosomatic Medicine and Psychotherapy, Central Institute of Mental Health, Mannheim, Germany. All women with BPD met DSM-IV diagnostic criteria for BPD as assessed by the International Personality Disorder Examination [26]. PTSD diagnosis and axis I comorbidity were assessed by the Structured Clinical Interview for DSM-IV Axis I Disorders (SCID-I [14]). A history of sexual traumatization in childhood, adolescence or adulthood was an inclusion criterion for all women with PTSD. A history of sexual traumatization was assessed by the Childhood Trauma Questionnaire for childhood and adolescence, and by the SCID-I and chart review for adulthood. In the group of women with PTSD, but without BPD, 18 (90\%) had suffered sexual traumatization before the age of 18 and eight $(40 \%)$ had a sexual trauma as adults. Among women with PTSD and BPD, all 15 had a history of sexual trauma before adulthood and six $(40 \%)$ had an additional sexual 
trauma as adults. In the group of women with BPD and without PTSD, $8(40 \%)$ had suffered a sexual trauma before the age of 18 and one woman as an adult. A lifetime psychotic or bipolar I disorder and a current substance dependence were exclusion criteria. Thirty-seven healthy women were recruited in Mannheim and included in the study, if careful screening did not detect a current or lifetime diagnosis of any axis I or II disorder. Trauma history information for healthy women was unavailable.

All 92 participants were females, between 18 and 50 years of age and gave written informed consent after procedures had been fully explained. The study was approved by the ethics committee of the Central Institute of Mental Health, Mannheim and was performed in accordance with the ethical standards of the 1964 Declaration of Helsinki. All subjects had German as their native language and had completed at least 9 years of school education. Sociodemographics and clinical data are reported in Table 1. Women with BPD showed similar rates of axis I comorbidity to women with PTSD. On average, severity of sexual abuse in childhood was significantly higher among women with PTSD, with or without comorbid BPD, than in women with BPD alone (Table 1). Severity of physical abuse did not differ between groups.

Self-report measures of disgust, anxiety, depression and childhood abuse

Disgust Sensitivity was assessed using Schienle's 37-item Questionnaire for the Assessment of Disgust Sensitivity (QADS [36], German version [37]), yielding a mean total score between 0 and 4 . Schienle and colleagues found mean scores of 2.23 in healthy women and 2.98 among women with BPD [37]. The State-Trait-AnxietyInventory (STAI-X2 [38], German version [24]) was used to measure trait-anxiety, with a sum score between 20 and 100. Depressive symptoms were assessed using the 21-item Beck Depression Inventory (BDI [3]) with a sum score from 0 to 63 . Scores greater than 10 are often considered to be of clinical relevance. The 28-item version of the Childhood Trauma Questionnaire (CTQ [4]) was used to assess severity of childhood abuse. Two subscales indicate the severity of physical and sexual abuse in childhood, both with a range of possible scores from 5 to 25 .

\section{Implicit association test}

The idea of the IAT is that verbal stimuli are classified more quickly when the target and attribute category pairings (e.g. Self/Disgust) match the individual's automatic associations with the target categories versus when the target and attribute category pairings are mismatched. For example, in our study a subject who implicitly associated herself more strongly with disgust than anxiety was expected to respond faster when the target concept "Self" and the attribute dimension "Disgust" were assigned to the same response key as compared to the pairing "Self" and "Anxiety".

During the IAT, subjects classified consecutive words into superordinate categories. The target categories were "Self" versus "Best Friend", the attribute categories were "Disgust" versus "Anxiety". In the centre of the screen, a series of consecutive words were presented that fell within the categories represented either on the left side or the right side of the screen (Fig. 1). The subject's task was to press the left response key to indicate that the word fell into the categories represented on the left side, and the right response key to indicate that the word fell into the categories on the right side. There was a total of five trial

Table 1 Sociodemographic and clinical variables across four groups

\begin{tabular}{|c|c|c|c|c|c|c|}
\hline & $\begin{array}{l}\text { Women with BPD } \\
\text { (without PTSD) } \\
(n=20)\end{array}$ & $\begin{array}{l}\text { Women with PTSD } \\
\text { (without BPD) } \\
(n=20)\end{array}$ & $\begin{array}{l}\text { Women with } \\
\text { BPD and PTSD } \\
(n=15)\end{array}$ & $\begin{array}{l}\text { Healthy } \\
\text { women } \\
(n=37)\end{array}$ & $F / \chi^{\mathrm{a}}$ & $P$ \\
\hline Age (years), $M(\mathrm{SD})$ & $27.6(7.1)$ & $32.8(8.1)$ & $32.1(9.7)$ & $31.0(8.3)$ & 1.53 & .21 \\
\hline High school degree & $50 \%$ & $35 \%$ & $27 \%$ & $49 \%$ & 7.97 & .24 \\
\hline Current major depression & $45 \%$ & $60 \%$ & $47 \%$ & - & 1.05 & .59 \\
\hline Current eating disorder & $35 \%$ & $30 \%$ & $53 \%$ & - & 2.11 & .35 \\
\hline Current anxiety disorder & $55 \%$ & $35 \%$ & $40 \%$ & - & 1.74 & .42 \\
\hline Current obsessive-compulsive disorder & $10 \%$ & $0 \%$ & $0 \%$ & - & 3.63 & .16 \\
\hline Current alcohol- or substance abuse & $35 \%$ & $15 \%$ & $40 \%$ & - & 3.13 & .21 \\
\hline Severity of physical abuse in childhood (CTQ), $M(\mathrm{SD})$ & $10.1(5.7)$ & $12.3(6.5)$ & $11.9(7.7)$ & - & 0.59 & .57 \\
\hline Severity of sexual abuse in childhood (CTQ), $M$ (SD) & $8.4(4.4)^{*}$ & $18.3(7.3)$ & $16.9(6.2)$ & - & 13.64 & $<.001$ \\
\hline
\end{tabular}

${ }^{a}$ Comparisons are analyses of variance for means, or chi-square tests for proportions, across each row

* Group mean significantly different from both other groups in post-hoc Scheffé tests $(P<.01)$ 


$\begin{array}{cc}\text { Press 'd' for } & \text { Press 'k' for } \\ \text { Disgust } & \text { Anxiety } \\ \text { or } & \text { or } \\ \text { Self } & \text { Best Friend } \\ & \\ & \\ & \text { disgusting }\end{array}$

Fig. 1 View of the computer screen representing a trial during a critical block of the Implicit Association Test. Subjects classify each stimulus word using either the d- or the k-key. In this example, the correct classification is on the d-key ('disgusting' $\rightarrow$ 'Disgust'). This block represents an associatively matched pairing for an implicitly disgust-prone individual (because Disgust and Self are matched). Across the trials of each block, the instructions and category pairings (upper left and right corner) remain unchanged. The verbal stimulus which needs to be categorized is always presented in the middle of the screen. Upon a key press, the next trial begins and a new verbal stimulus is presented (original stimuli in German and instructions given on the screen during the IAT can be obtained from the corresponding author)

blocks. In Blocks 1 and 2, the presented words had to be classified as an exemplar of either the target categories (e.g.; "she" $\rightarrow$ "Self" or "Best Friend") or the attribute categories (e.g., "anxious" $\rightarrow$ "Disgust" or "Anxiety"). Within each block, the target (or attribute) categories were assigned to one of two response keys. In Block 3, the first critical block, pairs of a target and an attribute category (e.g., "Self"/"Disgust" on the left, "Best Friend/Anxiety" on the right) were assigned to each of the two response keys. In Block 4, the key assignment of the target categories was switched when compared to Block 1 (e.g., Block 1: "Self" on the left, "Best Friend" on the right; Block 4: "Self" on the right, "Best Friend" on the left). In Block 5, the second critical block, the pairing between target and attribute categories was reversed compared to Block 3 (e.g.: Block 3-"Self/Disgust" and "Best Friend/ Anxiety"; Block 5: "Self/Anxiety" and "Best Friend/ Disgust").

The IAT is a measure of the relative strength of associations. Therefore, an equivalent comparison category for disgust was required. Anxiety was selected as a reference for disgust, since both are negative emotions. Thus, we controlled for the possibility that subjects may associate themselves with negative emotions in general, rather than with disgust in particular. We used the following stimuli for each category: For "Self" ("I") versus "Best Friend" ("She"), three additional personal stimuli (first name; last name; date of birth) were used. Because all our subjects were female, they were asked to choose a female best friend in order to avoid gender effects. For Disgust the stimuli were disgust, disgusting and nauseated; for Anxiety they were anxiety, fear, and anxious.

The IAT was administered on a PC and the order of blocks three and five was counterbalanced across subjects. The IAT score was calculated according to the improved scoring algorithm, resulting in a D-measure [19]. The D-measure represents the difference in reaction time between the critical blocks 3 and 5 divided by the standard deviation of all reaction times obtained during blocks 3 and 5. Negative values represent a stronger association between Self and Disgust (relative to Anxiety), while positive values indicate a stronger association between Self and Anxiety (relative to Disgust).

\section{Results}

Group differences in self-report measures

To test our first hypothesis that self-reported disgust sensitivity is higher in women with BPD or PTSD than in healthy women, we compared self-reported disgust sensitivity scores across four groups (Table 2; ANOVA: $d f=3$, partial $\eta^{2}=0.372$, Cohen's $f^{2}=0.97$ ). Disgust sensitivity did not differ significantly between women with BPD alone, PTSD alone, and women with BPD and PTSD. Because we did not find significant differences between diagnostic groups, we collapsed all 55 patients into one group and compared them with the healthy control group using t-tests for independent samples (Table 2, very right column; T-test for independent samples: Cohen's $d=1.41$ ). Disgust sensitivity was significantly higher among diagnosed than among healthy women. Levels of depression and trait anxiety showed a similar pattern, with patients being significantly more affected than controls (Table 2).

\section{Disgust-prone implicit self-concept}

To test our second hypothesis that Self is more strongly associated with Disgust (relative to Anxiety) among women with BPD and/or PTSD than in healthy women, we compared IAT scores across four groups (Table 2 and Fig. 2; ANOVA: $d f=3$, partial $\eta^{2}=0.137$, Cohen's $f^{2}=0.43$ ). On average, IAT-scores in diagnosed groups were negative, indicating that participants with BPD and/or PTSD implicitly associated themselves more strongly with disgust than with anxiety. IAT scores between the three groups of patients did not differ significantly. In contrast, mean IAT-scores of healthy women were positive, reflecting a stronger implicit association between Self and Anxiety than between Self and Disgust (Fig. 2). In post hoc tests, healthy women showed a significantly weaker 


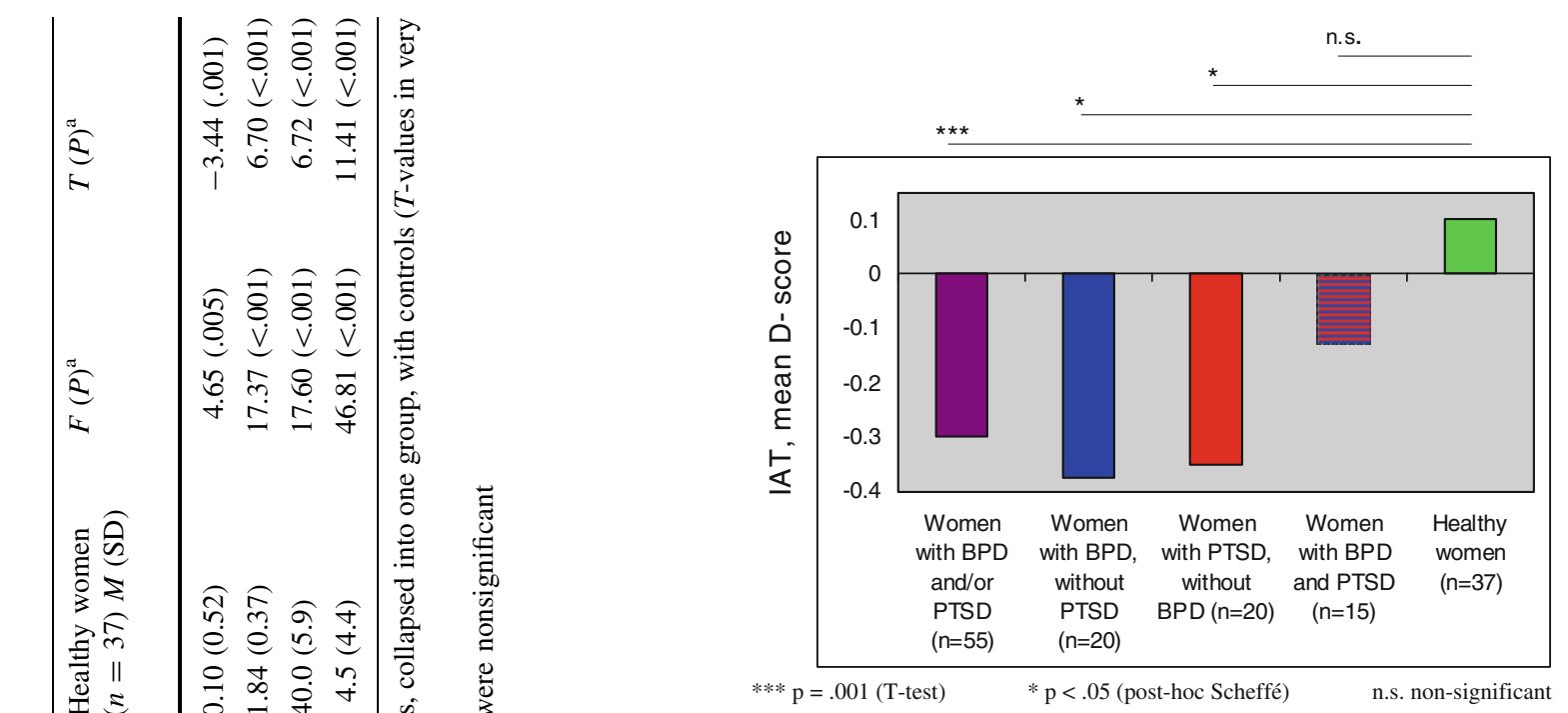

Fig. 2 Implicit Association Test of implicit disgust-prone selfconcept in women with BPD and/or PTSD as well as healthy women. More negative values indicate a more disgust-prone self-concept (relative to an anxiety-prone self-concept)

implicit Self-Disgust association than women with BPD alone and women with PTSD alone. IAT scores did not differ significantly between controls and women with BPD and PTSD. All 55 patients, collapsed into one group, exhibited a significantly more disgust-prone self-concept than controls (Table 2, very right column; $T$-test for independent samples: Cohen's $d=-0.73$ ).

We also determined how explicit self-report measures of disgust and anxiety were related to scores on the IAT among all 92 participants. To make questionnaire measures comparable to the IAT, which provides a relative measure of disgust- and anxiety-related implicit self-concept, we calculated a relative self-report index. For this purpose, both the trait anxiety and the disgust sensitivity self-report scores were z-standardized, and a constant of 3 was added so that all scores were greater than zero. Then a ratio of the standardized disgust and anxiety scores (i.e., QADS/STAI-X2) was calculated, which was unrelated to the IAT score $(r=$ $-0.10, P=.36$ ).

Correlations between childhood trauma and disgust

In the combined group of women with BPD and/or PTSD $(n=55)$, more severe physical abuse in childhood was associated with a less disgust-prone (and more anxietyprone) implicit self-concept (IAT, $r=.32, P=.02$ ). Severity of childhood physical abuse was not related to self-reported disgust sensitivity (QADS, $r=.15, P=.30$ ). Childhood sexual abuse severity was unrelated to disgustprone implicit self-concept (IAT, $r=.07, P=.62$ ) and explicit disgust sensitivity (QADS, $r=.06, P=.67$ ). 
Psychiatric comorbidity and disgust

To examine whether comorbid diagnoses of a current anxiety disorder, major depression, eating disorder or substance-related disorder affected self-reported disgust sensitivity (QADS) or implicit disgust-prone self-concept (IAT), we performed t-tests for independent samples among all 55 diagnosed participants. The 24 women with a current anxiety disorder (QADS: $M=2.71, \mathrm{SD}=0.42$; IAT: $M=-0.32, \mathrm{SD}=0.60)$ did not differ significantly from the 31 women without a current anxiety disorder (QADS: $M=2.49, \mathrm{SD}=0.72, T=1.34, P=.19$; IAT: $M=-0.28, \mathrm{SD}=0.55, T=-0.22, P=.83)$. Disgust variables did not differ between the 28 women with (QADS: $\quad M=2.61, \quad \mathrm{SD}=0.53 ; \quad \mathrm{IAT}: \quad M=-0.35$, $\mathrm{SD}=0.43$ ) when compared to the 27 women without a current major depression (QADS: $M=2.56, \mathrm{SD}=0.69$, $T=0.27, P=.79$; IAT: $M=-0.24, \mathrm{SD}=0.69, T=$ $-0.72, P=.48)$. The 21 participants with a current eating disorder (QADS: $M=2.52, \mathrm{SD}=0.53$; IAT: $M=-0.14$, $\mathrm{SD}=0.54)$ exhibited similar disgust-related responses to the 34 without a current eating disorder (QADS: $M=2.63$, $\mathrm{SD}=0.66, \quad T=-0.62, \quad P=.54 ; \quad \mathrm{IAT}: \quad M=-0.39$, $\mathrm{SD}=0.57, T=1.62, P=.11)$. Finally, among the 16 individuals with a current substance-related disorder (QADS: $\quad M=2.57, \quad \mathrm{SD}=0.72 ; \quad$ IAT: $\quad M=-0.37$, $\mathrm{SD}=0.54$ ) disgust levels were similar to the 39 without such a disorder (QADS: $M=2.60, \quad \mathrm{SD}=0.57, \quad T=$ $-0.17, P=.87$; IAT: $M=-0.27, \mathrm{SD}=0.58, T=-0.58$, $P=.56)$.

\section{Discussion}

Examining self-reported disgust sensitivity and implicit disgust-prone self-concept, we found elevated disgust in BPD and PTSD, independent of psychiatric comorbidity. Increased disgust sensitivity in PTSD provides empirical support for a recent conceptualization of disgust as a prominent emotion in this condition [10]. As far as BPD is concerned, our findings add disgust to a range of aversive emotions, such as anxiety, shame, guilt or anger [32], that contribute to emotional dysregulation [25, 34]. Our results further suggest that disgust is associated with BPD or PTSD at implicit-automatic as well as explicit levels. This finding and the fact that IAT scores were unrelated to selfreport measures are consistent with a view of disgust as a "noncognitive" emotion [28] that may partly act outside conscious awareness or control. Implicit associations are thought to represent automatic structures in memory and to underlie maladaptive cognitive schemata. Through the influence of implicit cognitive schemata on both perception and behaviour, persons with a disgust-prone self-concept may be more vulnerable to disgust-related stimuli and to emotional dysregulation. A stronger implicit disgust-related self-concept in BPD and PTSD has implications for psychotherapeutic treatments, both from a cognitivebehavioural [25] and a psychodynamic perspective [21]. In these patients with traumatic experiences, disgust may play an important role besides shame, anxiety or anger, and psychotherapists should try to address pathological disgust that may operate outside conscious awareness or control. From a behavioural perspective, mirror confrontation with one's own body (which is often an object of disgust) and desensitization procedures are used successfully in inpatient dialectical behaviour therapy [5]. The same applies to dialectical behaviour treatment of PTSD, where discrimination training between trauma-related disgust memories and current reality plays an important role [39].

Contrary to our hypothesis, we did not find a correlation between disgust measures and severity of sexual abuse in childhood. There are three possible explanations for this negative finding that should be examined in future studies. First, disgust measures in this study did not specifically assess trauma- or abuse-related aspects of disgust; second, the level of disgust experienced during traumatization was not assessed; and third, due to the limited sample size this may be a false negative finding. With respect to implicit measures, sexual abuse may affect implicit associations of Self with both Anxiety and Disgust. The IAT being a relative measure of association strengths, these effects may cancel each other out. We did, however, find more severe physical childhood abuse to be associated with a more anxiety-prone (relative to disgust-prone) implicit self-concept, possibly due to intense fear experienced during physical abuse.

Limitations of our study need to be considered. First, only women were included in our study and possible gender differences remain unclear. Second, sample sizes were limited. Therefore, the unexpected finding that women with both BPD and PTSD did not show a significantly more disgust-prone implicit self-concept than controls could either be a false negative finding or speak against a straightforward additive effect of BPD-PTSD comorbidity on disgust measures. Third, conclusions based on the IAT are restricted since it is a relative index of disgust- and anxiety-prone implicit self-concept and does not provide an absolute measure of the implicit association between self and a single attribute concept. Future investigations should examine IAT variants other than our Self/ Best Friend-Disgust/Anxiety design as well as IATs that target trauma-relevant disgust domains rather than disgust in general. Similarly, our self-report measure of disgust sensitivity may have underestimated the level of disgust first because respondents may be hesitant to disclose negative emotions and second because the questionnaire did 
not focus on trauma-related disgust and respective stimuli. These measurement issues, both for indirect and direct measures, need to be addressed in future research. Fourth, longitudinal studies could investigate whether disgust and implicit self-concept change over time, since implicit fear was shown to be amenable to change during therapy [42]. Fifth, in addition to disgust sensitivity in response to external stimuli, as assessed in our study, future studies should investigate self-reported self-disgust in traumarelated disorders [30] as well as disgust in PTSD following nonsexual trauma. Finally, explicit and implicit aspects of disgust between trauma-related and other disorders such as depression or schizophrenia should be investigated to determine the specificity of our findings for trauma-related disorders [36].

In summary, our study provides empirical evidence of increased disgust in BPD and PTSD at explicit and implicit levels. Future research should examine disgust in traumarelated disorders, its relation to psychopathology, other emotions such as shame or anger and to social functioning, and to develop psychotherapeutic strategies that tackle explicit as well as implicit aspects of pathological disgust.

Acknowledgments We are grateful to all participants. Nicolas Rüsch was supported by a Marie Curie Outgoing International Fellowship of the European Union. Preliminary data from a subgroup of participants were presented at the 23rd Annual Meeting of the International Society for Traumatic Stress Studies, 16 November 2007, Baltimore.

Conflict of interest The authors declare that they have no conflict of interest.

\section{References}

1. Barrett LF, Niedenthal PM, Winkielman P (2005) Emotion and consciousness. Guilford Press, New York

2. Beck AT, Freeman A, Davis DD (2004) Cognitive therapy of personality disorders. Guilford Press, New York

3. Beck AT, Steer RA (1987) Beck depression inventory manual. Psychological Corporation, San Antonio

4. Bernstein DP, Stein JA, Newcomb MD, Walker E, Pogge D, Ahluvalia T, Stokes J, Handelsman L, Medrano M, Desmond D, Zule W (2003) Development and validation of a brief screening version of the childhood trauma questionnaire. Child Abuse Negl 27:169-190

5. Bohus M, Haaf B, Simms T, Limberger MF, Schmahl C, Unckel C, Lieb K, Linehan MM (2004) Effectiveness of inpatient dialectical behavioral therapy for borderline personality disorder: a controlled trial. Behav Res Ther 42:487-499

6. Buchheim A, George C, Liebl V, Moser A, Benecke C (2007) Mimische Affektivität von Patientinnen mit einer BorderlinePersönlichkeitsstörung während des Adult Attachment Projective. Zeitschrift für Psychosomatische Medizin und Psychotherapie 53:339-354

7. Catani C, Adenauer H, Keil J, Aichinger H, Neuner F (2009) Pattern of cortical activation during processing of aversive stimuli in traumatized survivors of war and torture. Eur Arch Psychiatry Clin Neurosci 259:340-351

8. Charash M, McKay D (2002) Attention bias for disgust. J Anxiety Disord 16:529-541

9. Charash M, McKay D, Dipaolo N (2006) Implicit attention bias for disgust. Anxiety Stress Coping 19:353-364

10. Dalgleish T, Power MJ (2004) Emotion-specific and emotionnon-specific components of posttraumatic stress disorder (PTSD): implications for a taxonomy of related psychopathology. Behav Res Ther 42:1069-1088

11. Darwin C (1965) The expression of the emotions in man and animals (Original publication 1872; John Murray, London). University of Chicago Press, Chicago

12. Davey GCL, Buckland G, Tantow B, Dallos R (1998) Disgust and eating disorders. Eur Eat Disord Rev 6:201-211

13. Fiori M (2009) A new look at emotional intelligence: a dualprocess framework. Pers Soc Psychol Rev 13:21-44

14. First MB, Spitzer RL, Gibbon M, Williams JBW (1997) Structured clinical interview for DSM-IV axis I disorders (SCID-I), clinician version. American Psychiatric Association Press, Washington, DC

15. Foy DW, Sipprelle RC, Rueger DB, Carroll EM (1984) Etiology of posttraumatic stress disorder in Vietnam veterans: analysis of premilitary, military, and combat exposure influences. J Consult Clin Psychol 52:79-87

16. Gawronski B, Bodenhausen GV (2006) Associative and propositional processes in evaluation: an integrative review of implicit and explicit attitude change. Psychol Bull 132:692-731

17. Greenwald AG, Farnham SD (2000) Using the implicit association test to measure self-esteem and self-concept. J Pers Soc Psychol 79:1022-1038

18. Greenwald AG, McGhee DE, Schwartz JL (1998) Measuring individual differences in implicit cognition: the implicit association test. J Pers Soc Psychol 74:1464-1480

19. Greenwald AG, Nosek BA, Banaji MR (2003) Understanding and using the implicit association test: I. An improved scoring algorithm. J Pers Soc Psychol 85:197-216

20. Greenwald AG, Poehlman TA, Uhlmann E, Banaji MR (2009) Understanding and using the implicit association test: III. Meta-analysis of predictive validity. J Pers Soc Psychol 97: $17-41$

21. Hartocollis $P$ (1978) Time and affects in borderline disorders. Int J Psychoanal 59:157-163

22. Hofmann W, Gawronski B, Gschwendner T, Le H, Schmitt M (2005) A meta-analysis on the correlation between the implicit association test and explicit self-report measures. Personal Soc Psychol Bull 31:1369-1385

23. Koenen KC, Nugent NR, Amstadter AB (2008) Gene-environment interaction in posttraumatic stress disorder: review, strategy and new directions for future research. Eur Arch Psychiatry Clin Neurosci 258:82-96

24. Laux L, Glanzmann P, Schaffner P, Spielberger CD (1981) Das State-Trait-Angstinventar. Beltz, Weinheim

25. Linehan MM (1993) Cognitive-behavioral treatment of borderline personality disorder. The Guilford Press, New York

26. Loranger AW, Sartorius N, Andreoli A, Berger P, Buchheim P, Channabasavanna SM, Coid B, Dahl A, Diekstra RF, Ferguson B (1994) The international personality disorder examination: the world health organization/alcohol, drug abuse, and mental health administration international pilot study of personality disorders. Arch Gen Psychiatry 51:215-224

27. McKay D, Olatunji BO (2009) Disgust and psychopathology: next steps in an emergent area of treatment and research. In: Olatunji BO, McKay D (eds) Disgust and its disorders: theory, assessment and treatment implications. American Psychological Association, Washington, DC, pp 285-292 
28. McNally RJ (2002) Disgust has arrived. J Anxiety Disord 16:561-566

29. Olatunji BO, Sawchuk CN (2005) Disgust: characteristic features, social manifestations, and clinical implications. J Soc Clin Psychol 24:932-962

30. Overton PG, Markland FE, Taggart HS, Bagshaw GL, Simpson J (2008) Self-disgust mediates the relationship between dysfunctional cognitions and depressive symptomatology. Emotion 8:379-385

31. Phillips ML, Senior C, Fahy T, David AS (1998) Disgust-the forgotten emotion of psychiatry. Br J Psychiatry 172:373-375

32. Power MJ, Tarsia M (2007) Basic and complex emotions in depression and anxiety. Clin Psychol Psychother 14:19-31

33. Rozin P, Haidt J, McCauley C (2009) Disgust: the body and soul emotion in the 21 st century. In: Olatunji BO, McKay D (eds) Disgust and its disorders: theory, and treatment implications. American Psychological Association, Washington, DC, pp 9-29

34. Rüsch N, Lieb K, Göttler I, Hermann C, Schramm E, Richter H, Jacob GA, Corrigan PW, Bohus M (2007) Shame and implicit self-concept in women with borderline personality disorder. Am J Psychiatry 164:500-508

35. Rüsch N, Todd AR, Bodenhausen GV, Olschewski M, Corrigan PW (2010) Automatically activated shame reactions and perceived legitimacy of discrimination: a longitudinal study among people with mental illness. J Behav Ther Exp Psychiatry 41:60-63

36. Schienle A, Schäfer A, Stark R, Walter B, Franz M, Vaitl D (2003) Disgust sensitivity in psychiatric disorders: a questionnaire study. J Nerv Ment Dis 191:831-834
37. Schienle A, Walter B, Stark R, Vaitl D (2002) Ein Fragebogen zur Erfassung der Ekelempfindlichkeit (FEE). Zeitschrift für Klinische Psychologie und Psychotherapie 31:110-120

38. Spielberger CD, Gorsuch RL, Lushene RE (1970) Manual for the state-trait-anxiety-inventory. Consulting Psychologists Press, Palo Alto

39. Steil R, Dyer A, Priebe K, Kleindienst N, Bohus M (2010) Dialectical behavior therapy for posttraumatic stress disorder related to childhood sexual abuse: a pilot study of an intensive residential treatment program. J Trauma Stress (in press)

40. Teachman BA (2006) Pathological disgust: in the thoughts, not the eye, of the beholder. Anxiety Stress Coping 19:335-351

41. Teachman BA, Gregg AP, Woody SR (2001) Implicit associations for fear-relevant stimuli among individuals with snake and spider fears. J Abnorm Psychol 110:226-235

42. Teachman BA, Woody SR (2003) Automatic processing in spider phobia: implicit fear associations over the course of treatment. J Abnorm Psychol 112:100-109

43. Troop NA, Murphy F, Bramon E, Treasure JL (2000) Disgust sensitivity in eating disorders: a preliminary investigation. Int $\mathrm{J}$ Eat Disord 27:446-451

44. Zanarini MC (2000) Childhood experiences associated with the development of borderline personality disorder. Psychiatr Clin North Am 23:89-101 